\section{Insulin resistance might link obesity and congestive heart failure}

In a prospective trial carried out in Sweden, Ingelsson et al. found that insulin resistance is a predictive risk factor for congestive heart failure (CHF). Obesity and diabetes are already established risk factors for CHF, but the potential link between insulin resistance and CHF had not previously been investigated.

The Uppsala Longitudinal Study of Adult Men (ULSAM) included 1,187 men who were $\geq 70$ years old at the time of enrollment during 1990-1995, and were free of CHF and valvular disease. Several baseline examinations were performed, including the euglycemic insulin clamp technique for testing insulin sensitivity. The participants were followed until the end of 2002, and of the 1,187 men, 104 had definitive CHF.

The study showed that insulin resistance predicted CHF incidence independently of diabetes, obesity and other established risk factors, although it is possible that impaired glucose regulation might have been a sign of impending diabetes.

Obesity was also associated with $\mathrm{CHF}$, but the association was no longer significant when clamp glucose rate disposal was included in the multivariate analysis. This finding suggests that insulin resistance might be the causal pathway between obesity and CHF; nevertheless, the authors note that trial data can not "... definitively disentangle the causative relations between obesity, insulin resistance and CHF". Further research into this area is warranted.

Rachel Murphy

Original article Ingelsson E et al. (2005) Insulin resistance and risk of congestive heart failure. JAMA 294:334-341

\section{Ankle-brachial index values and vascular risk}

The ankle-brachial index (ABI) is the ratio of the ankle and brachial systolic blood pressures. An $A B I$ of less than 0.90 indicates the presence of peripheral vascular disease.

Doobay and Anand have sought to establish the validity of a low $\mathrm{ABI}$ as a predictor of future cardiovascular events. Their systematic review included prospective studies that used
$\mathrm{ABI}$ values of between 0.80 and 0.90 to classify patients as having peripheral arterial disease and recorded the incidence of cardiovascular events. Of nine studies included in the metaanalysis, seven were population cohorts of relatively healthy individuals and two studies included cohorts with suspected peripheral arterial disease. Data were combined using a random-effects model that accounted for within-study and between-study variation.

$\mathrm{ABI}$ predicted incident coronary heart disease with high specificity (92.7\%) but low sensitivity (16.5\%). For incident stroke the figures were similar (92.2\% and 16\% respectively), although for cardiovascular mortality specificity was slightly lower (87.9\%) and sensitivity higher (41.0\%). Among population-based cohorts, then, $A B I$ is a highly specific but insensitive predictor of future cardiovascular events, which makes it unlikely to be useful as a generic screening test. While a low $A B I$ could help to identify a high-risk patient, a normal value cannot exclude risk of cardiovascular events without further tests. As a simple, noninvasive and inexpensive test, however, it is recommended as part of the vascular risk assessment for selected individuals.

Claire Braybrook

Original article Doobay AV and Anand SS (2005) Sensitivity and specificity of the ankle-brachial index to predict future cardiovascular outcomes: a systematic review. Arterioscler Thromb Vasc Biol 25: 1463-1469

\section{Hypertrophic obstructive cardiomyopathy: a comparison of treatment options}

EBM Asymmetrical septal hypertrophy that causes a left-ventricular outflow tract (LVOT) gradient is a common feature of hypertrophic obstructive cardiomyopathy (HCOM). Septal myectomy is the standard treatment for patients with this condition, although percutaneous transluminal septal myocardial ablation (PTSMA) is a nonsurgical alternative that involves injecting alcohol into a septal branch of the left anterior descending artery, causing local ischemia and myocardial infarction. Both of these techniques are effective methods to reduce septal thickness and thus LVOT gradients in patients with HCOM. After septal myectomy, there is an immediate decrease in LVOT gradient, whereas the gradient 